\title{
Painéis de pínus e bagaço de cana empregando- se dois adesivos para uso na construção civil
}

\author{
Pine and sugarcane bagasse particleboard using two \\ adhesives for use in the construction industry
}

\section{Ana Laura Soler Cunha Buzo \\ Estéfani Suana Sugahara \\ Sérgio Augusto de Mello da Silva \\ Elen Aparecida Martines Morales \\ Maximiliano dos Anjos Azambuja}

\section{Resumo}

${ }^{1}$ Ana Laura Soler Cunha Buzo ${ }^{1}$ Universidade Estadual Paulista J úlio de Mesquita Filho Ilha Solteira - SP - Brasil https:// orcid. org/ 0000-0001-8566-5527

${ }^{2}$ Estéfani Suana Sugahara ${ }^{2}$ Universidade Estadual Paulista J úlio de Mesquita Filho Guaratinguetá - SP - Brasil

${ }^{3}$ Sérgio Augusto de Mello da Silva
${ }^{\text {Universidade Estadual Paulista J úlio }}$
Ilha Solteira - SP - Brasil

${ }^{4}$ Elen Aparecida Martines Morales 4 Universidade Estadual Paulista Itapeva - SP - Brasil

${ }^{5}$ Maximiliano dos Anjos Azambuja 5Universidade Estadual Paulista J úlio de Mesquita Filho Bauru - SP - Brasil

Recebido em 26/06/18 Aceito em 19/01/19

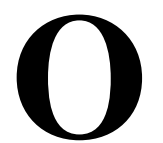

presente trabalho objetivou produzir e avaliar painéis aglomerados empregando-se partículas de bagaço de cana-de-açúcar (Saccharum officinarum) e de pínus (Pinus taeda, Pinus elliottii), comparando-se a eficiência de dois adesivos, ureia-formaldeído e poliuretano derivado do óleo de mamona. Os painéis foram preparados com $60 \%$ de partículas de pínus e com $40 \%$ de partículas de bagaço de cana. Foram avaliadas propriedades físicas (densidade, teor de umidade e inchamento em espessura após 24 h de imersão em água) e mecânicas (módulo de elasticidade, módulo de resistência à flexão estática e tração perpendicular às faces) dos painéis de acordo com os requisitos de uso das normas NBR 14810-1 (ABNT, 2013a), NBR 14810-2 (ABNT, 2013b) e EN-312. Os painéis confeccionados com poliuretano de mamona apresentaram maior eficiência, sendo compatíveis com os requisitos físicos e mecânicos das normas brasileiras e europeia para painéis do tipo P6 (painéis estruturais para uso em condições severas de carga, em condições secas). Os painéis produzidos com ureia-formaldeído são compatíveis com painéis classificados como P2 (painéis não estruturais para uso em condições secas). Dessa forma, os resultados alcançados comprovaram a eficácia dos painéis produzidos, que podem ser empregados nas indústrias moveleira e da construção civil.

Palavras-chave: Painéis aglomerados. Poliuretano derivado do óleo de mamona. Ureiaformaldeído. Propriedades físico-mecânicas.

\section{Abstract}

This research project aimed to produce and evaluate particleboard using sugarcane bagasse (Saccharum officinarum) and pínus (Pinus taeda; Pinus elliotti) particles by comparing the efficiency of the two adhesives: ureaformaldehyde and polyurethane adhesive based on castor oil. The panels were made with $60 \%$ of pine wood particles, and $40 \%$ of sugarcane bagasse particles. The physical properties (density, moisture content and thickness swelling after $24 \mathrm{~h}$ in water immersion), and mechanical properties (modulus of elasticity, modulus in static bending, and perpendicular traction to faces) of the panels were evaluated according to the use requirements set by the standards NBR 14810-1 (ABNT, 2013a), NBR 14810-2 (ABNT, 2013b) and $E N-312$. Panels produced with $P U$-Castor presents higher efficiency and compatibility with the physical and mechanical requirements of Brazilian and European standards for panels of type P6 (structural panels for use under severe load conditions in dry conditions). Panels produced with ureaformaldehyde are compatible with panels classified as P2 (non-structural panels for use in dry conditions). The results achieved proved the effectiveness of the panels produced, which can be used in the furniture and construction industries.

Keywords: Agglomerated panels. Polyurethane based on castor oil. Urea-formaldehyde polymer. Physical-mechanical properties.

BUZO, A. L. S. C.; SUgahARA, E. S.; SILVA, S. A. de M. da; MORALES, E. A. M.; AZAMBUJ A, M. dos A. Painéis de pínus 183 e bagaço de cana empregando-se dois adesivos para uso na construção civil. Ambiente Construído, Porto Alegre, v. 19, n. 4, p. 183-193, out./ dez. 2019.

ISSN 1678-8621 Associação Nacional de Tecnologia do Ambiente Construído.

http:// dx. doi. org/ 10.1590/ s1678-86212019000400350 


\section{Introdução}

A madeira é uma das matérias-primas mais antigas e possui características amplamente aproveitadas pela humanidade. Entretanto, a excessiva exploração das florestas nativas, sem preocupação com a reposição florestal e sem o emprego de planos de manejo, gerou impactos irreversíveis ao meio ambiente. Aumentar as áreas de florestas plantadas é uma proposta para amenizar esses impactos, que, devido às oportunidades de ampliar o manejo florestal, atende às maiores demandas e, consequentemente, contribui com as ações sustentáveis (SOCIEDADE..., 2009).

Outro fator que contribuiu com os danos ambientais foi a geração de resíduos agroindustriais, pois eles apresentam valores significativos e são, por muitos, simplesmente queimados ou dispensados em locais inadequados. Dessa forma, passou-se a considerar sua utilização na cadeia produtiva industrial (ALBERTINI; CARMO; PRADO FILHO, 2007). Além da contribuição ambiental, o aproveitamento de resíduos objetivando agregar valor ao produto é uma solução viável para a fabricação de derivados de madeira, como é o caso dos painéis aglomerados (ALVES; SILVA, 2014).

Conforme dados da Food and Agriculture Organization of the United Nations (FOOD..., 2016), a produção mundial de painéis à base de madeira em 2016 atingiu $387.639 .000 \mathrm{~m}^{3}$. Segundo Klímek et al. (2018), considerando os altos volumes de produção juntamente com as restrições evidenciadas dos recursos naturais, uma futura escassez no fornecimento de madeira está potencialmente se tornando um assunto crítico. Desse modo, a variedade crescente de recursos lignocelulósicos pode ser de importância estratégica, pois a utilização de resíduos agrícolas como matéria-prima certamente tem benefícios econômicos, reduz os encargos ambientais e melhora a eficiência da cadeia de valor.

Portanto, a produção de painéis derivados de madeira com reaproveitamento de resíduos de diferentes origens pode contribuir para suprir a demanda de painéis e estimular a produção de novos materiais, bem como a utilização de resíduos lignocelulósicos, que contribui para amenizar os impactos ambientais causados pelos resíduos se depositados a céu aberto (CARASCHI et al., 2009).

\section{Referencial teórico}

De acordo com a Confederação Nacional da Indústria (CONFEDERAÇÃO..., 2017), desde os anos 2000 a produção nacional de painéis de madeira tem registrado crescimento anual médio de $6,4 \%$.
Esse crescimento com relação à produção e ao consumo, tanto mundialmente, quanto nacionalmente, ocorre devido à facilidade nas aplicações para diversos fins e à quantidade de produtos disponíveis (BERTOLINI, 2011). Esse tipo de painel encontra utilizações na construção civil para aplicações estruturais ou não estruturais (pisos, forros, paredes, esquadrias, portas, telhados, andaimes, formas de concreto, etc.), na construção de barcos (partes estruturais e decorativas), na fabricação de móveis, instrumentos musicais, embalagens industriais, caixas e outros (FIORELLI et al., 2014).

Nos últimos anos as indústrias de construção e móveis exigiram painéis aglomerados com alta durabilidade e características sustentáveis (KUSUMAH et al., 2017). Dessa maneira, os estudos com painéis priorizaram as altas propriedades mecânicas e a substituição da madeira por outros materiais lignocelulósicos, uma vez que a composição química dos materiais lignocelulósicos é semelhante à da madeira, preservando, assim, nossos recursos naturais (CRAVO et al., 2015).

Frequentemente, segundo Silva et al. (2017), as madeiras reflorestadas não têm atingido os requisitos para painéis de partículas estruturais. Com isso, em vez de aumentar as áreas de reflorestamento, considerou-se substituir as madeiras por resíduos agroindustriais, pois, além de a geração de resíduos lignocelulósicos das agroindústrias brasileiras, como casca de café, sabugo de milho, casca de arroz, casca de amendoim, casca de coco, casca de mamona e bagaço de cana-de-açúcar ser considerada promissora como fonte da matéria-prima para atender à acrescente demanda de mercado de painéis aglomerados, reduzir o consumo de madeira de reflorestamento contribui para a redução do custo de produção desses painéis particulados (SILVA et al., 2017).

De acordo com Mendes et al. (2010), qualquer material lignocelulósico pode, em princípio, ser utilizado para a confecção de painéis de partículas. Melo et al. (2014) fabricaram painéis com madeira (Eucalyptus grandis), bambu (Bambusa vulgaris) e/ou casca de arroz (Oryza sativa), combinados ou não. Os resultados indicaram que os aglomerados fabricados com bambu apresentaram melhor qualidade do que aqueles que utilizam casca de arroz como matéria-prima. Para a maioria dos parâmetros, os painéis de partículas fabricados exclusivamente com partículas de madeira apresentaram desempenho semelhante aos painéis de partículas de bambu e madeira-bambu. 
De acordo com Caraschi et al. (2009), muitos resíduos produzidos por agroindústrias podem ser utilizados para fazer painéis de partículas. Entre os resíduos agrícolas, o bagaço de cana-de-açúcar tem se destacado devido ao fato de sua composição química mostrar similaridade com a de madeiras moles (SILVA et al., 2017) e em virtude de a quantidade gerada representar no Brasil um dos mais significativos resíduos sólidos gerados na agroindústria sucroalcooleira (PEDRESCHI, 2009).

Mendes et al. (2010), Freire et al. (2011), Tabarsa et al. (2011) e Mendes et al. (2012) já avaliaram o uso do bagaço de cana na produção de aglomerados e compararam suas qualidades com painéis comerciais produzidos com madeira de eucalipto e pínus. Concluíram que painéis confeccionados com bagaço de cana são muito promissores para a produção de mobiliário.

Varanda (2016) produziu painéis de partículas homogêneas de alta densidade, com resíduos de madeira de Pinus elliottii e casca de aveia (Avena sativa) aderidos sob pressão com dois tipos de adesivo, poliuretano à base de óleo de mamona e melamina formaldeído, nos percentuais de $11 \%$ e $13 \%$, e avaliou o desempenho físico-mecânico de tais painéis para aplicação em pisos com base na norma NBR 14810 e em diversas normas relacionadas a pisos de madeira. Os resultados apontaram propriedades físico-mecânicas dos painéis em alguns tratamentos superiores aos requisitos estipulados por normas nacionais e internacionais. Quanto ao desempenho para pisos, os painéis apresentaram desempenho semelhante a três espécies de madeira na maioria das propriedades avaliadas, o que evidencia a potencialidade dos painéis produzidos para aplicação na indústria de pisos.

Oliveira et al. (2016) avaliaram painéis industriais de MDP (painel de partículas de média densidade) feitos de eucalipto e pínus por duas empresas brasileiras e um painel de MDP industrial feito com bagaço de cana-de-açúcar produzido na China. Analisaram propriedades físicas (umidade, densidade, absorção e inchamento em espessura após 2 h e 24 h de água) e propriedades mecânicas (módulo de elasticidade, módulo de ruptura, adesão interna, arranchamento de parafuso e testes de dureza de Janka). Constataram que os painéis feitos com bagaço de cana apresentaram propriedades físicas e mecânicas comparáveis ou superiores às produzidas com eucalipto e pínus.

De acordo com Nascimento et al. (2015), uma das principais preocupações da indústria de painéis derivados de madeira é a busca por adesivos de menor impacto ambiental. Considerando a indústria de painéis aglomerados, a maior parte utiliza adesivos sintéticos, especialmente à base de formaldeído, substância altamente tóxica e liberada no meio ambiente durante o processo de prensagem a quente. Nesse contexto surgem novas propostas de adesivos como o poliuretano à base de mamona, adesivo altamente resistente à água se comparado à ureia formaldeído e ainda tem origem renovável por meio da utilização da mamona, planta abundante no Brasil, da qual se extrai o óleo de mamona a partir da semente da planta Ricinus communis (CANGEMI et al., 2006).

Visto que o bagaço de cana-de-açúcar, um resíduo agroindustrial gerado em abundância no Brasil, mostra-se como material promissor para a fabricação de painéis de partículas, Fiorelli et al. (2013) produziram painéis aglomerados com partículas de bagaço de cana-de-açúcar utilizando adesivo poliuretano à base de óleo de mamona. De acordo com os autores, as propriedades mecânicas obtidas mostraram-se satisfatórias para uso na construção civil e agrícola, bem como em casas, edifícios agrícolas e aplicações estruturais.

Varanda et al. (2013) produziram e avaliaram painéis de partículas de Eucalyptus grandis e casca de aveia (Avena sativa) em diferentes porcentagens, aglomerados sob pressão com adesivo PU-Mamona (poliuretano bicomponente derivado do óleo de mamona) na proporção de $10 \%$ em relação à massa seca das partículas. Pela análise de variância (ANOVA), a fração de partículas de Eucalyptus grandis foi significativa apenas no módulo de resistência à flexão estática (MOR), e painéis produzidos com $100 \%$ de casca de aveia apresentaram maior valor de MOR.

Santos et al. (2014) avaliaram o potencial de uso dos resíduos de madeira tauari (Couratari oblongifolia) como matéria-prima para a produção de painéis de partículas usando adesivo de poliuretano bicomponente à base de óleo de mamona em um teor de $16 \%$. Os resultados indicaram que o painel pode ser classificado como um aglomerado de alta densidade.

Apesar das ótimas propriedades físico-mecânicas mostradas pelo adesivo PU-Mamona, seu custo ainda é muito superior ao dos adesivos empregados em painéis. Porém, além de haver a tendência por produtos com apelo ambiental e de origem renovável, o adesivo PU-Mamona, quando utilizado na produção de painéis de madeira, pode ter seu custo reduzido, pois não necessita de altas temperaturas de prensagem se comparado às temperaturas exigidas para as resinas à base de formaldeído, colocando-o, assim, em nível competitivo para produções comerciais (BERTOLINI et al., 2013). O emprego do adesivo 
PU-Mamona também suprime o uso da parafina (necessária ao se aplicar adesivo à base de ureiaformaldeído para reduzir a higroscopicidade dos painéis), o que colabora com a redução dos custos.

Nesse sentido, o presente trabalho apresenta a produção e a avaliação de painéis aglomerados compostos de partículas de bagaço de cana-deaçúcar (Saccharum officinarum) e partículas de madeira de pínus (Pinus taeda, Pinus elliottii) utilizando-se e comparando a eficiência de dois tipos de adesivos, o poliuretano bicomponente derivado de óleo de mamona (PU-Mamona) e a ureia-formaldeído (UF).

\section{Método}

Neste estudo utilizou-se bagaço de cana-de-açúcar (Saccharum officinarum) coletado em usinas sucroalcooleiras da região noroeste do Estado de São Paulo e partículas de madeira das espécies Pinus taeda e Pinus elliotti coletadas em serrarias da cidade de Ilha Solteira, SP.

O material coletado foi seco ao sol, e posteriormente se realizou uma seleção manual para a retirada de corpos estranhos e inadequados para a produção dos painéis, evitando, assim, problemas no processo de trituração no moinho de facas e na compactação dos painéis, e principalmente problemas como formação de bolhas no interior do painel e possíveis delaminações.

Em seguida, todo o bagaço e as partículas de pínus foram peneirados para adequação das dimensões das partículas, que, segundo Alves (2013), é de 2 $\mathrm{mm}$ a $6 \mathrm{~mm}$.

Dessa forma, o material utilizado para confecção dos painéis com adesivo PU-Mamona foi seco ao sol até atingir teor de umidade em torno de $10 \%$ e o material para confecção dos painéis de UF, foi seco em estufa à temperatura de $60{ }^{\circ} \mathrm{C}$ até atingir teor de umidade em torno de $3 \%$, valor compatível com o sugerido pelos fabricantes dos adesivos.

As partículas de bagaço de cana e de pínus foram classificadas quanto a sua granulometria por meio de uma adaptação do ensaio para a determinação da composição granulométrica de agregados miúdos e graúdos para concreto, proposto pela NM 248 (ABNT, 2003), no qual se utilizou um agitador de partículas com sistema vibratório. O ensaio possibilitou determinar o módulo de finura da mistura de partículas de acordo com as porcentagens retidas de material em cada peneira do conjunto.

Os painéis foram produzidos com dimensões de 35 cm x $35 \mathrm{~cm}$ x $1 \mathrm{~cm}$ e densidade nominal de 0,67 g.cm ${ }^{-3}$. Em seguida, calculou-se a massa das partículas para confecção dos painéis de acordo com as porcentagens definidas para os tratamentos propostos.

Foram propostos dois tratamentos $\left(\mathrm{T}_{B}\right.$ e $\left.\mathrm{T}_{\mathrm{D}}\right)$, ambos com $60 \%$ de partículas de madeira de pínus, $40 \%$ de partículas de bagaço e $10 \%$ de resina em relação à massa seca das partículas, sendo diferenciados pelo tipo de adesivo. Os tratamentos propostos para confecção dos painéis estão apresentados na Tabela 1.

Para a confecção dos painéis, inicialmente se realizou a adição manual da resina UF e/ou do adesivo PU-Mamona, e depois se utilizou um processo mecânico por meio de um misturador de tambor.

Os painéis feitos com PU-Mamona tiveram o adesivo utilizado na proporção de 1:1 entre poliol e pré-polímero, o qual foi misturado ao substrato em duas etapas. Na primeira etapa adicionou-se e homogeneizou-se o poliol, composto oriundo do óleo de mamona, e na segunda etapa adicionou-se e homogeneizou-se o pré-polímero, composto oriundo do petróleo, porque, segundo Silva e Lahr (2007), esse procedimento propicia melhor homogeneização do adesivo poliuretano bicomponente ao substrato.

Nos painéis produzidos com resina UF, adotaramse os procedimentos feitos por Eleoterio (2000), utilizando-se emulsão de parafina na proporção de $1 \%$ de sólidos da emulsão em relação à massa das fibras com o objetivo de reduzir a higroscopicidade final dos painéis. Foi adicionado também sulfato de amônia na dosagem de 2,5\% de sólidos de catalizador em relação ao teor de sólidos da resina e água para promover a dissolução dos materiais e atingir o teor de umidade desejado para o colchão de partículas homogêneas.

Após a adição e a homogeneização do adesivo às partículas, iniciou-se o processo para preparação do colchão de partículas, realizando-se uma préprensagem com pressão de compactação de 0,4 $\mathrm{MPa}$, com o intuito de conferir à massa de partículas uma forma inicial que auxiliou no processo de compactação e confecção dos painéis de partículas aglomeradas.

A Figura 1 ilustra o processo de preparação do colchão de partículas, em que se efetuaram as etapas de acomodação das partículas na formadora de colchão (Figura 1a), pré-prensagem manual aplicando-se força de 5 t (Figura 1b) e colchão de partículas pronto para ser compactado em prensa com controle de temperatura e pressão (Figura 1c).

Posteriormente, iniciou-se a prensagem dos painéis, executada através da prensa hidráulica com pressão aferida em $50 \mathrm{MPa}$, temperatura de $100{ }^{\circ} \mathrm{C}$ para o 
adesivo PU-Mamona e de $130{ }^{\circ} \mathrm{C}$ para a resina UF, e duração de $10 \mathrm{~min}$ de prensagem, com tempo inicial de 3 min e intervalos para alívio de pressão de $30 \mathrm{~s}$, objetivando-se evitar a concentração de gases no interior dos painéis. Na Figura 2 ilustramse a compactação de um painel na prensa (Figura 2a) e um painel logo após a prensagem (Figura 2b).

Esse procedimento foi utilizado para produzir quatro painéis para cada tratamento, dos quais foram obtidos 10 corpos de prova para avaliação e caracterização de suas propriedades através dos ensaios físicos e mecânicos, conforme especifica a NBR 14810-2 (ABNT, 2013b).

As propriedades físicas e mecânicas avaliadas foram densidade aparente $(\rho)$, teor de umidade $(U)$, inchamento em espessura após 24 h de imersão em água (I), módulo de elasticidade (MOE), módulo de ruptura em flexão estática (MOR) e resistência à tração perpendicular às faces (TP), conforme preconiza a norma brasileira NBR 14810-2 (ABNT, 2013b). Além da norma brasileira, utilizou-se a norma EN 312 (EUROPEAN..., 2003) com o intuito de se avaliarem as propriedades e classificar os painéis fabricados. A Tabela 2 apresenta os valoreslimite das propriedades físicas e mecânicas estabelecidos pelos documentos normativos utilizados.

Os tipos de painéis apresentados pela Tabela 2 são: painéis do tipo $\mathrm{P} 2$, que segundo a norma brasileira NBR 14810-2 (ABNT, 2013b) são "Painéis não estruturais para uso em condições secas”; painéis do tipo P4, que tanto na norma brasileira NBR 148102 (ABNT, 2013b) quanto na norma europeia EN 312 (EUROPEAN..., 2003) referem-se a "Painéis estruturais para uso em condições secas”; e painéis do tipo P6, que para ambas as normas condizem a "Painéis estruturais para uso em condições severas de carga em condições secas”.

Após os ensaios físicos e mecânicos dos painéis realizou-se uma análise estatística descritiva dos resultados levando-se em consideração as médias, os coeficientes de variação e os intervalos de confiança.

\section{Resultados e discussões}

A Tabela 3 apresenta uma análise estatística descritiva dos resultados obtidos das propriedades físicas (densidade aparente, teor de umidade e inchamento por $24 \mathrm{~h}$ ) dos painéis confeccionados com UF e dos painéis confeccionados com PUMamona, sendo $\bar{X}$ a média amostral, IC 95\% o intervalo de confiança de 95\%, que indica a probabilidade de $95 \%$ de o intervalo conter o verdadeiro valor da média, e $C V$ o coeficiente de variação, que em estatística é o parâmetro utilizado para expressar a variabilidade dos dados em relação à média tirando a influência da ordem de grandeza da variável, ou seja, quanto menor o $C V$, mais homogêneo é o conjunto de dados.

Tabela 1 - Tratamentos propostos para confecção dos painéis

\begin{tabular}{c|c|c|c}
\hline Tratamento & Madeira (\%) & Bagaço (\%) & Adesivo \\
\hline $\mathrm{T}_{\mathrm{B}}$ & 60 & 40 & PU-Mamona \\
$\mathrm{T}_{\mathrm{D}}$ & 60 & 40 & UF \\
\hline
\end{tabular}

Figura 1 - Preparação do colchão de partículas

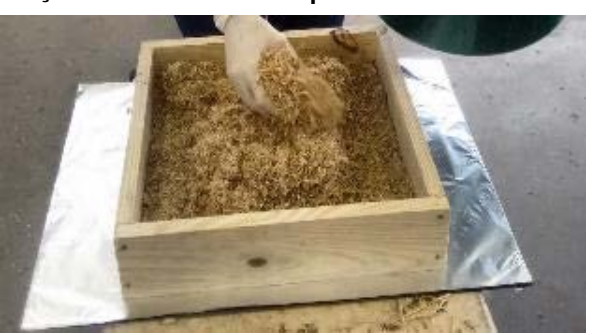

(a)

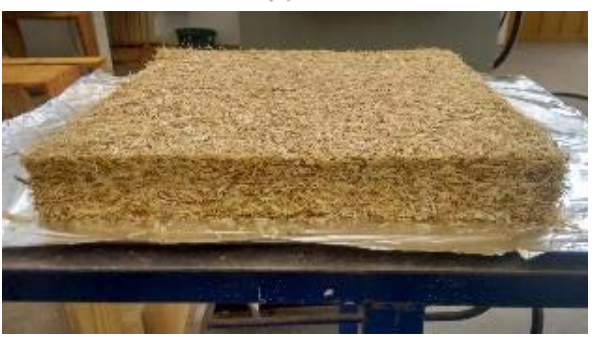

(c)

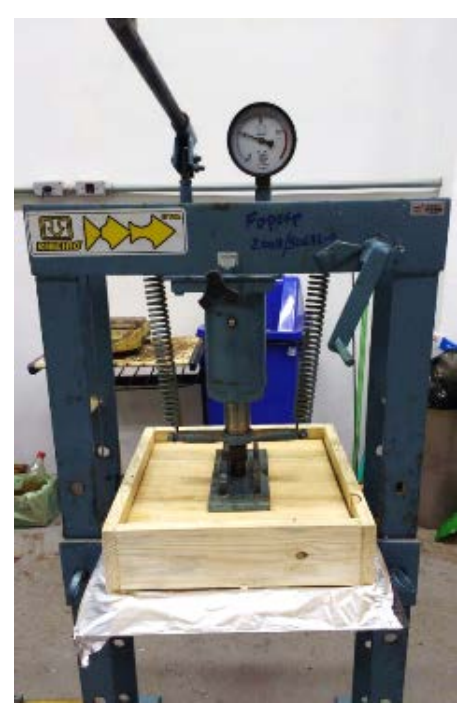

(b) 
Figura 2 - Prensagem do painel

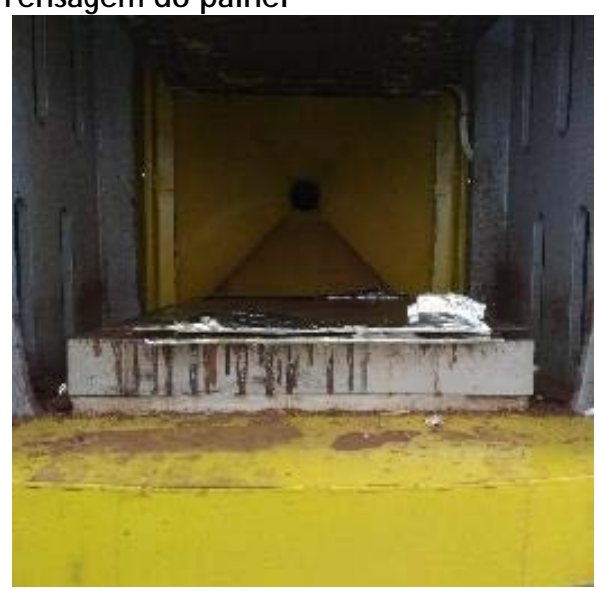

(a)

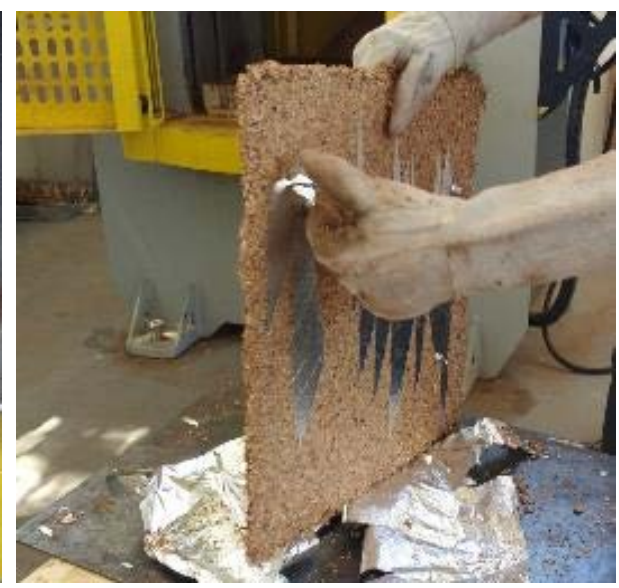

(b)

Tabela 2 - Parâmetros normativos

\begin{tabular}{|c|c|c|c|c|c|c|c|}
\hline Normas & $\begin{array}{c}\text { Tipo de } \\
\text { painel }\end{array}$ & $\rho\left(\mathrm{g} / \mathrm{cm}^{3}\right)$ & I (\%) & U (\%) & $\begin{array}{l}\text { MOE } \\
\text { (MPa) } \\
\end{array}$ & $\begin{array}{l}\text { MOR } \\
\text { (MPa) } \\
\end{array}$ & $\begin{array}{c}\text { TP } \\
(\mathrm{MPa}) \\
\end{array}$ \\
\hline \multirow{2}{*}{$\begin{array}{c}\text { EN } 312 \\
\text { (EUROPEAN..., } \\
\text { 2003) }\end{array}$} & P4 & - & 16 & 5 a 13 & 2.300 & 16 & 0,4 \\
\hline & P6 & - & 15 & 5 a 13 & 3.150 & 20 & 0,6 \\
\hline \multirow{3}{*}{$\begin{array}{l}\text { NBR 14810-2 } \\
\text { (ABNT, 2013b) }\end{array}$} & $\mathrm{P} 2$ & 0,51 a 0,75 & 18 & 5 a 13 & 1.800 & 11 & 0,4 \\
\hline & $\mathrm{P} 4$ & 0,51 a 0,75 & 19 & 5 a 13 & 2.300 & 16 & 0,4 \\
\hline & P6 & 0,51 a 0,75 & 16 & 5 a 13 & 3.150 & 20 & 0,6 \\
\hline
\end{tabular}

Tabela 3 - Resultados das propriedades físicas

\begin{tabular}{|c|c|c|c|c|c|c|}
\hline \multirow{2}{*}{$\begin{array}{l}\text { Propriedades } \\
\text { físicas }\end{array}$} & \multicolumn{3}{|c|}{ UF } & \multicolumn{3}{|c|}{ PU-Mamona } \\
\hline & $\bar{x}$ & $C V(\%)$ & IC 95\% & $\bar{X}$ & $C V(\%)$ & IC $95 \%$ \\
\hline$\rho\left(\mathrm{g} / \mathrm{cm}^{3}\right)$ & 935 & 4 & 906 а 965 & 1060 & 2 & 1.038 a 1.083 \\
\hline U (\%) & 6,4 & 3,3 & 6,2 a 6,6 & 8,4 & 0,8 & 8,4 a 8,5 \\
\hline I (\%) & 62,6 & 7,6 & 58,3 а 66,9 & 6,8 & 19,9 & 5,6 а 7,9 \\
\hline
\end{tabular}

Com relação à densidade, segundo a NBR 14810-1 (ABNT, 2013a), painéis com densidades entre 551 e $750 \mathrm{~kg} / \mathrm{m}^{3}$ são definidos como painéis de partículas de média densidade. Assim, os resultados obtidos na Tabela 3 indicaram que ambos os tratamentos em análise encontram-se com valores de densidade acima dos de referência. Observa-se também que o coeficiente de variação dos tratamentos está dentro dos limites estipulados tanto pela norma brasileira NBR 14810-2 (ABNT, 2013b), < 7\%, quanto pela norma europeia EN 312 (EUROPEAN..., 2003), < 10\%.

Quanto aos valores obtidos na determinação do teor de umidade, constatou-se que os valores médios, assim como os valores dos intervalos de confiança dos dois tratamentos, encontram-se dentro do intervalo recomendado pelas normas brasileira e europeia (5\% a 13\%), conforme apresentado na Tabela 2.
Ainda sobre o teor de umidade, observa-se que o valor encontrado $(U=6,4 \%)$ nos painéis produzidos com adesivo UF é compatível com as exigências da NBR 14810-2 (ABNT, 2014b). Segundo Oliveira et al. (2016), estudos realizados em painéis MDP produzidos por indústrias brasileiras (painéis comerciais nacionais) apresentaram teor de umidade de $10,9 \%$, ou seja, superior ao valor determinado nos estudos desenvolvidos neste trabalho, mas ainda dentro dos requisitos normativos. A umidade pode ter sofrido influência de vários fatores, como o material e a umidade das partículas, a porcentagem de adesivo utilizado, a temperatura de prensagem e a utilização de aditivos como a emulsão de parafina, sem prejuízo ao painel produzido.

Com relação aos valores obtidos no ensaio de inchamento em espessura após 24 h, para a média e para o intervalo de confiança, observa-se através da Tabela 2 que os painéis confeccionados com a 
resina PU-Mamona atenderam aos requisitos determinados para painéis do tipo P6 (painéis estruturais para uso em condições severas de carga em ambientes secos), conforme estabelecido pela NBR 14810-2 (ABNT, 2013b) e pela norma europeia (16\% e 15\% respectivamente). Já os painéis confeccionados com resina UF ficaram muito acima do máximo estabelecido pelas referências normativas.

Nota-se também que os valores encontrados para inchamento em espessura dos painéis de UF (I = $62,6 \%)$ são maiores que os valores encontrados por Barros Filho et al. (2011) para painéis aglomerados fabricados com $50 \%$ de bagaço e $50 \%$ de pínus e com teor de $9 \%$ de resina UF (I = 33,2\%). É válido ressaltar que, de acordo com a norma brasileira, o valor máximo recomendado para o inchamento em espessura após 24 h é de $18 \%$, ou seja, ambos os estudos desenvolvidos ultrapassaram as exigências normativas e confirmaram a baixa estabilidade dimensional dos painéis produzidos com a resina UF. Segundo Dias (2005), isso se justifica porque a baixa resistência à umidade de painéis aglomerados produzidos com resina UF limita seu emprego ao uso em condições secas.

Com relação à análise estatística, há o coeficiente de variação $(C V)$ para indicar se a análise foi precisa, pois se adotou a classificação de Ferreira (1991) em relação à precisão do processo. Se o $C V$ for menor que $15 \%$, tem-se uma ótima precisão amostral com baixa dispersão dos dados; se o $C V$ estiver entre $15 \%$ e $20 \%$, tem-se boa precisão com média dispersão dos dados; se estiver entre $20 \%$ e $30 \%$, entende-se que a amostra tem uma precisão regular com alta dispersão dos dados; e se o $C V$ der acima de 30\%, significa que a amostra tem uma precisão ruim com uma dispersão muito alta. Consequentemente é possível verificar, de acordo com as análises apresentadas na Tabela 3, que os coeficientes de variação são baixos, ou seja, os valores da caracterização das propriedades físicas dos painéis são precisos.

Quanto às propriedades mecânicas (módulo de elasticidade e módulo de resistência na flexão estática e resistência à tração perpendicular às faces), a Tabela 4 apresenta uma análise estatística descritiva dos resultados obtidos dos painéis confeccionados com UF e dos painéis confeccionados com PU-Mamona, sendo $\bar{X}$ a média amostral, $c v$ o coeficiente de variação e IC 95\% o intervalo de confiança de $95 \%$.

Na Tabela 4 verifica-se que o valor do MOR obtido no ensaio de flexão estática dos painéis confeccionados com UF atende aos padrões mínimos do documento normativo brasileiro (11 $\mathrm{MPa}$ ). Já o valor dos painéis com poliuretano propiciou resistência muito acima das mínimas estabelecidos pela NBR e pela norma europeia, sendo aproximadamente 2,3 vezes maior do que o MOR dos painéis confeccionados com UF.

Nota-se também que o valor do MOR obtido neste trabalho para os painéis de ureia (15 $\mathrm{MPa})$ está acima dos valores encontrados por Mendes (2008) para aglomerados com bagaço de cana-de-açúcar e UF $(\mathrm{MOR}=9,74 \mathrm{MPa})$ e bem acima dos valores encontrados por Barros Filho et al. (2011) para painéis aglomerados com $50 \%$ de bagaço e $50 \%$ de pínus e resina UF (MOR = 3,42 MPa). Quanto aos painéis com poliuretano, Dias (2005) apresentou para aglomerados fabricados com resina poliuretana à base de mamona valores para o módulo de resistência à flexão estática $(\mathrm{MOR}=18 \mathrm{MPa})$ bem inferiores aos valores encontrados neste trabalho $(\mathrm{MOR}=35 \mathrm{MPa})$

Com relação ao resultado do módulo de elasticidade (MOE) dos painéis de ureia, observa-se que a média superou o mínimo determinado para painéis do tipo P4 (2.300 MPa). Dessa forma, sua classificação levando como referência apenas o MOE é de painéis do tipo P4 (painéis estruturais para uso em condições secas) de acordo com a NBR 14810-2 (ABNT, 2013b) e com a EN 312 (EUROPEAN..., 2003). Já os painéis de PU-Mamona obtiveram tanto a média quanto o mínimo do intervalo de confiança muito acima daqueles determinados pelas normas utilizadas para análise (Tabela 2).

Tabela 4 - Resultados das propriedades mecânicas

\begin{tabular}{c|c|c|c|c|c|c}
\hline \multirow{2}{*}{$\begin{array}{c}\text { Propriedades } \\
\text { mecânicas }\end{array}$} & \multicolumn{3}{|c|}{ UF } & \multicolumn{3}{c}{ PU-Mamona } \\
\cline { 2 - 7 } & $\overline{\boldsymbol{X}}$ & $\boldsymbol{C V} \mathbf{( \% )}$ & IC 95\% & $\overline{\boldsymbol{X}}$ & $\boldsymbol{C V} \mathbf{( \% )}$ & IC 95\% \\
\hline MOR (MPa) & 15 & 19 & 12 a 18 & 35 & 10 & 31 a 39 \\
MOE (MPa) & 2416 & 20 & 1913 a 2919 & 3555 & 13 & 3159 a 3951 \\
TP $(\mathrm{MPa})$ & 1,14 & 6,2 & 1,07 a 1,20 & 2,23 & 11,4 & 2,02 a 2,43 \\
\hline
\end{tabular}


Ainda sobre os valores encontrados de MOE, percebeu-se que o valor dos painéis produzidos com a resina UF está maior que os valores encontrados por Mendes (2008) para painéis aglomerados com bagaço de cana e UF (MOE $=661 \mathrm{MPa})$ e maior que os valores encontrados por Barros Filho et al. (2011) para painéis aglomerados com 50\% de bagaço e $50 \%$ de pínus e resina UF (MOE $=710$ $\mathrm{MPa})$. O valor dos painéis de PU-Mamona também superou os valores encontrados por Dias (2005) para painéis de madeira aglomerada fabricados com resina poliuretana à base de mamona $(\mathrm{MOE}=3.034$ $\mathrm{MPa})$.

Quanto à resistência à tração perpendicular, nota-se que tanto as médias quanto os intervalos de confiança de ambos os tratamentos superaram o mínimo preconizado pela NBR 14810-2 (ABNT, 2013b) e pela norma europeia EN 312 (EUROPEAN..., 2003) para painéis do tipo P6 (painéis estruturais para uso em condições severas de carga em ambientes secos).

Observa-se também que os valores obtidos para resistência à tração perpendicular para os painéis de UF foram superiores aos encontrados por Barros Filho et al. (2011) para painéis aglomerados fabricados com $50 \%$ de bagaço e $50 \%$ de pínus e com teor de $9 \%$ de resina UF (TP $=0,53 \mathrm{MPa})$. Com relação aos painéis de PU-Mamona, os valores encontrados para TP também são bem superiores aos encontrados por Poleto et al. (2013) para painéis de madeira aglomerada com $50 \%$ de pínus e $50 \%$ de eucalipto e adesivo PU-Mamona (TP = 0,9 MPa).

Com as análises apresentadas na Tabela 4, é possível afirmar que os valores alcançados para as propriedades mecânicas dos painéis produzidos com PU-Mamona são homogêneos, ou seja, têm baixa dispersão de valores em torno das médias avaliadas. Porém, verifica-se que os valores dos coeficientes de variação do MOR e do MOE dos painéis produzidos com UF estão na faixa entre $15 \%$ e $20 \%$, ou seja, apesar de terem boa precisão, possuem uma média dispersão dos dados. Assim, pode-se concluir através dos resultados das propriedades mecânicas que a resina PU-Mamona é mais estável e ainda proporcionou melhores resultados nas propriedades avaliadas.

Os altos valores obtidos para os ensaios na flexão estática (MOR e MOE) e em relação à tração perpendicular às faces podem estar relacionados a uma adequada distribuição granulométrica das partículas utilizadas. Para Pierre (2010), o desempenho dos painéis é, em grande parte, o reflexo das características geométricas da partícula (comprimento e espessura), que influem fortemente na transmissão das propriedades da madeira ao painel, em que partículas longas e finas dão origem a painéis com maior resistência à flexão estática e estabilidade dimensional, ao mesmo tempo em que painéis produzidos com partículas curtas e espessas melhoram sua resistência à tração perpendicular. A área superficial específica da partícula também influencia nas propriedades por se tratar da área de contato entre as partículas e a linha de cola.

\section{Conclusões}

Com base nas análises realizadas, confirma-se a viabilidade de produção de painéis aglomerados com $60 \%$ de partículas de madeira de pínus e $40 \%$ de partículas de bagaço de cana-de-açúcar empregando-se $10 \%$ dos adesivos PU-Mamona e UF em relação à massa de partículas. Dessa forma, é possível indicar o aproveitamento de resíduos para a produção de painéis de partículas aglomeradas de alta densidade, pois, em sua maioria, as propriedades físicas e mecânicas investigadas foram compatíveis com os limites estipulados pelos documentos normativos brasileiro, NBR 14810-2 (ABNT, 2013b), e europeu EN 312 (EUROPEAN..., 2003).

Quanto aos aglutinantes, o PU-Mamona apresentou melhores resultados para as propriedades físicas e mecânicas quando comparado à resina UF, destacando-se como uma das principais vantagens desse adesivo a alta resistência à umidade, apontada através dos resultados do ensaio de inchamento. Além de apresentar um excelente desempenho para as propriedades analisadas, o adesivo PU-Mamona destaca-se por sua eficiência energética, pois necessita de menores temperaturas de prensagem em comparação à UF e por ser possível atingir o teor de umidade desejado para as partículas passando apenas por secagem ao sol, ou seja, dispensando o uso de estufa. É importante citar também que se trata de um material de origem renovável, pois a mamona é planta abundante no Brasil e o adesivo não emite vapores de formaldeído, que são poluentes.

Analisando ainda os aglutinantes, pode-se observar através dos resultados obtidos que, para a resina UF, a propriedade de inchamento não atingiu o valor mínimo preconizado pelas normas NBR 14810-2 (ABNT, 2013b) e EN 312 (EUROPEAN..., 2003), porém os resultados obtidos para todas as outras propriedades, tanto físicas como mecânicas (densidade aparente, teor de umidade, módulo de elasticidade, módulo de ruptura em flexão estática e resistência à tração perpendicular às faces), comprovam seu atendimento aos requisitos normativos para painéis aglomerados, o que também é compatível com os resultados encontrados na literatura. 
Nesse contexto evidencia-se que o aproveitamento de resíduos e o uso de materiais alternativos, no caso o bagaço de cana-de-açúcar e a resina PUMamona, além da contribuição ambiental, mostram-se como uma alternativa viável para a fabricação de painéis aglomerados com propriedades próximas ou superiores às dos painéis disponíveis comercialmente, podendo ser utilizados com sucesso na produção moveleira e em várias aplicações na construção civil.

Por fim, de acordo com os documentos normativos de referência, pode-se classificar os painéis produzidos com PU-Mamona como "Painéis estruturais para uso em condições severas de carga em condições secas (P6)”.

Em trabalhos futuros sugere-se utilizar outras proporções de adesivo para verificar a correlação entre a eficiência e a porcentagem utilizada, principalmente se observando e comparando a resistência ao inchamento.

\section{Referências}

ALBERTINI, S.; CARMO, L. F.; PRADO

FILHO, L. G. Utilização de Serragem e Bagaço de Cana-de-Açúcar para Adsorção de Cádmio.

Ciência e Tecnologia de Alimentos, v. 27, n. 1, p. 113-118, 2007.

ALVES, L. S. Aproveitamento de Resíduos de Empresas Moveleiras da Região de São José do Rio Preto para Confecção e Avaliação de Painéis Aglomerados. Ilha Solteira, 2013. 78 f. Dissertação (Mestrado em Engenharia Civil) Curso de Engenharia Civil, Universidade Estadual Paulista “Júlio de Mesquita Filho”, Ilha Solteira, 2013.

ALVES, L. S.; SILVA, S. A. M. Confecção e Avaliação de Chapas de Madeira Aglomerada Homogêneas Empregando-se Resíduos de Empresas Moveleiras de São José do Rio Preto e Poliuretana Derivada de Óleo de Mamona. In: ENCONTRO BRASILEIRO DE MADEIRAS E DE ESTRUTURAS DE MADEIRA, 14., Natal, 2014. Anais... Natal: Ebramem, 2014.

\section{ASSOCIAÇÃO BRASILEIRA DE NORMAS}

TÉCNICAS. NBR NM 248: agregados: determinação da composição granulométrica. Rio de Janeiro, 2003.

ASSOCIAÇÃO BRASILEIRA DE NORMAS TÉCNICAS. NBR 14810-1: painéis de partículas de média densidade: parte 1: Terminologia. Rio de Janeiro, 2013a.
ASSOCIAÇÃO BRASILEIRA DE NORMAS TÉCNICAS. NBR 14810-2: painéis de partículas de média densidade: parte 2: requisitos e métodos de ensaio. Rio de Janeiro, 2013b.

BARROS FILHO, R. M. et al. Hybrid Chipboard Panels Based on Ugarcane Bagasse, Urea Formaldehyde and Melamine Formaldehyde Resin. Industrial Crops And Products, v. 33, n. 2, p. 369-373, 2011.

BERTOLINI, M. S. Emprego de Resíduos de Pinus sp Tratado Com Preservantes CCB na Produção de Chapas de Partículas Homogêneas Utilizando Resina Poliuretana à Base de Mamona. São Carlos, 2011. 128 f. Dissertação (Mestrado em Engenharia Civil) - Escola de Engenharia, Instituto de Física, Instituto de Química, Universidade de São Paulo, São Carlos, 2011.

BERTOLINI, M. S. et al. Accelerated Artificial Aging of Particleboards From Residues of CCB Treated Pinus sp and Castor Oil Resin. Materials Research, v. 16, n. 2, p. 293-303, 2013.

CANGEMI, J. M. et al. Study of the Biodegradation of a Polymer Derived From Castor Oil by Scanning Electron Microscopy, Thermogravimetry and Infrared Spectroscopy. Polímeros: Ciência e Tecnologia, v. 16, n. 2, p. 129-135, 2006.

CARASCHI, J. C. et al. Avaliação de Painéis Produzidos a Partir de Resíduos Sólidos Para Aplicação na Arquitetura. Polímeros: Ciência e Tecnologia, v. 19, n. 1, p. 47-53, 2009.

CONFEDERAÇÃO NACIONAL DA INDÚSTRIA. Florestas Plantadas: oportunidades e desafios da indústria de base florestal no caminho da sustentabilidade. Brasília, 2017.

CRAVO, J. C. M. et al. Manufacture of Particleboard Based on Cement Bag and Castor Oil Polyurethane Resin. Construction And Building Materials, v. 87, n. 1, p. 8-15, 2015.

DIAS, F. M. Aplicação de Resina Poliuretana à Base de Mamona na Fabricação de Painéis de Madeira Compensada e Aglomerada. São Carlos, 2005. 158 f. Tese (Doutorado em Engenharia Civil) - Escola de Engenharia, Universidade de São Paulo, São Carlos, 2005.

ELEOTERIO, J. R. Propriedades Físicas e Mecânicas de Painéis MDF de Diferentes Densidades e Teores de Resina. Piracicaba, 2000. 122 f. Dissertação (Mestrado em Engenharia Civil) - Curso de Ciência e Tecnologia de Madeiras, Universidade de São Paulo, Piracicaba, 2000. 
EUROPEAN COMMITTEE FOR

STANDARDIZATION. EN 312: particleboards: specifications. Bruxelas, 2003.

FERREIRA, P. V. Estatística Experimental Aplicada à Agronomia. Maceió: Edufal, 1991.

FOOD AND AGRICULTURE ORGANIZATION OF THE UNITED NATIONS. Forest Products: yearbook annuaire: forest products 2010-2014. Rome: Forestry Policy and Resources Division Fao, 2016.

FIORELLI, J. et al. Physico-Chemical and Anatomical Characterization of Residual Lignocellulosic Fibers. Cellulose, v. 21, n. 5, p. 3269-3277, 2014.

FIORELLI, J. et al. Sugarcane Bagasse and Castor Oil Polyurethane Adhesive-Based Particulate Composite. Materials Research, v. 16, n. 2, p. 439-446, jan. 2013.

FREIRE, C. S. et al. Physical Properties of Commercial Particle-Boards Made of Sugarcane Bagasse and Wood. Floresta e Ambiente, v. 18, p. 178-185, 2011.

KLÍMEK, P. et al. Utilizing Miscanthus Stalks as Raw Material For Particleboards. Industrial Crops and Products, v. 111, p. 270-276, jan. 2018.

KUSUMAH, S. S. et al. Utilization of Sweet Sorghum Bagasse and Citric Acid For Manufacturing of Particleboard II: influences of pressing temperature and time on particleboard properties. Journal of Wood Science, v. 63, p. 161-172, 2017.

MELO, R. R. et al. Physical and Mechanical Properties of Particleboard Manufactured From Wood, Bamboo and Rice Husk. Materials Research, v. 17, n. 3, p. 682-686, 2014.

MENDES, R. F. Qualidade de Painéis Aglomerados Produzidos na China Utilizando Bagaço de Cana. In: ENCONTRO BRASILEIRO EM MADEIRAS E EM ESTRUTURAS DE MADEIRA, 11., Londrina. Anais... Londrina, 2008.

MENDES, R. F. et al. Particleboards Produced With Sugar Cane Bagasse and Eucalyptus Wood. Scientia Forestalis, v. 38, p. 285-295, 2010.

MENDES, R. F. et al. Determination of the Elastic Modulus of Particleboard by Stress Wave Timer. Floresta e Ambiente, v. 19, p. 117-122, 2012.

NASCIMENTO, M. F. et al. Painéis OSB Fabricados Com Madeiras da Caatinga do Nordeste do Brasil. Ambiente Construído, Porto Alegre, v. 15, n. 1, p. 41-48, jan./mar. 2015.
OLIVEIRA, S.L. et al. Particleboard Panels Made From Sugarcane Bagasse: characterization for use in the furniture industry. Materials Research, v. 19, p. 914-922, 2016.

PEDRESCHI, R. Aproveitamento do Bagaço de Cana da Indústria Sucroalcooleira na Produção de Painéis Aglomerados. Lavras, 2009. 49 f. Tese (Doutorado em Ciência e Tecnologia da Madeira) - Universidade Federal de Lavras, Lavras, 2009.

PIERRE, F. C. Caracterização Físico-Mecânica de Painéis Aglomerados de Eucalyptus grandis Com Adição de Resíduos Industriais Madeireiros. Botucatu, 2010. 134 f. Tese (Doutorado em Agronomia) - Faculdade de Ciências Agronômicas Universidade Estadual Paulista, Botucatu, 2010.

POLETO, S. F. S. et al. Produção de Painéis de Partículas Homogêneas (PPH) Utilizando Resíduos de Espécies de Reflorestamento. São Carlos: Ed. EESC/USP, 2013.

SANTOS, W. L. F. et al. Particleboard Manufactured From Tauari (Couratarioblongifolia) Wood Waste Using Castor Oil Based Polyurethane Resin. Materials Research, v. 17, n. 3, p. 657-663, 2014.

SOCIEDADE BRASILEIRA DE SILVICULTURA. O Papel das Florestas Plantadas Para Atendimento das Demandas Futuras da Sociedade. In: CONGRESSO FLORESTAL MUNDIAL, 13., Buenos Aires, 2009. Anales... Buenos Aires, 2009.

SILVA, M. R. et al. Hybrid Sandwich Particleboard Made With Sugarcane, Pinus taeda Thermally Treated and Malva Fibre From Amazon. Materials Research, v. 21, n. 1, 2017.

SILVA, S. A. M.; LAHR, F. A. R. Painéis de Partículas Confeccionadas Com Resíduos de Madeiras Tropicais de Baixa Densidade. In: RECICLAGEM de Resíduos para a construção civil. Belo Horizonte: Editora da UFBH, 2007.

TABARSA, T. et al. Evaluation of Surface Roughness and Mechanical Properties of Particleboard Panels Made From Bagasse. Composites Part B: Engineering, v. 42, p. 13301335, 2011.

VARANDA, L. D. et al. Oat Hulls as Addition to High Density Panels Production. Materials Research, v. 16, n. 6, p. 1355-1361, 2013.

VARANDA, L. D. Painéis de Alta Densidade Para Aplicação em Pisos: produção e avaliação de desempenho. São Carlos, 2016. 171 f. Tese (Doutorado em Engenharia Civil) - Escola de Engenharia, Universidade de São Paulo, São Carlos, 2016. 


\section{Agradecimentos}

Os autores agradecem ao Programa de PósGraduação da Faculdade de Engenharia de Ilha
Solteira, da Universidade Estadual Paulista, e à Capes, pelo apoio prestado ao desenvolvimento deste trabalho.

Ana Laura Soler Cunha Buzo

Departamento de Engenharia Civil | Universidade Estadual Paulista J úlio de Mesquita Filho | Alameda Bahia, 550, Zona Norte | Ilha Solteira - SP - Brasil | CEP 15385-000 | Te.: (18) 3745-1137 | E-mail: analaurasolercunha@gmail.com

\section{Estéfani Suana Sugahara}

Faculdade de Engenharia | Universidade Estadual Paulista J úlio de Mesquita Filho | Av. Dr. Ariberto Pereira da Cunha, 333, Portal das Colinas | Guaratinguetá - SP - Brasil | CEP 12516-410 | Tel.: (12) 3123-2814 | E-mail: esugahara@fef.edu.br

\section{Sérgio Augusto de Mello da Silva}

Departamento de Engenharia Civil | Universidade Estadual Paulista Júlio | E-mail: sams@dec.feis.unesp.br

\section{Elen Aparecida Martines Morales}

Campus Experimental de Itapeva | Universidade Estadual Paulista | Rua Geraldo Alckimin, 519, Vila Nossa Senhora de Fátima | Itapeva SP - Brasil | CEP 18409-010 | Tel.: (15) 3524-9100 | E-mail: elen@itapeva.unesp.br

\section{Maximiliano dos Anjos Azambuja}

Departamento de Engenharia Civil e Ambiente | Universidade Estadual Paulista Júlio de Mesquita Filho | Av. Eng. Luiz Edmundo C. Coube 14-01, Vargem Limpa | Bauru - SP - Brasil | CEP 17033-360 | Tel.: (14) 3103-6000 | E-mail: maximilianoazam@feb.unesp.br

\section{Revista Ambiente Construído}

Associação Nacional de Tecnologia do Ambiente Construído

Av. Osvaldo Aranha, 99 - 3o andar, Centro

Porto Alegre - RS - Brasil

$$
\text { CEP } 90035-190
$$

Telefone: +55 (51) 3308-4084

Fax: +55 (51) 3308-4054

www. seer. ufrgs. br/ ambienteconstruido

E-mail: ambienteconstruido@ufrgs.br 